\title{
Malignant lymphoma and extensive viral wart formation in a patient with intestinal lymphangiectasia and lymphocyte depletion
}

\author{
M. WARD \\ M.B., M.R.C.P. \\ W. P. SMall \\ V.R.D., M.B., Ch.B., Ch.M., F.R.C.S.(Eng), \\ F.R.C.P.(Eng.)
}

AnNe Le RouX*

B.Sc., M.B., Ch.B.

W. SIRCUS

M.D., Ph.D., F.R.C.P.(Lond.), F.R.C.P.(Ed.)

Gastro-intestinal Unit and *Department of Pathology, Western General Hospital, Edinburgh

\begin{abstract}
Summary
A patient is described, the subject of a previous report (Ross et al., 1971), who subsequently developed gross peripheral mosaic warts and a malignant small bowel lymphoma in association with marked lymphocyte depletion.
\end{abstract}

\section{Introduction}

It is known that peripheral lymphocytopenia accompanies intestinal lymphangiectasia (Strober et al., 1967) and that this is due to an excessive loss of lymphocytes from the gut (Roberts and Douglas, 1976). Although this lymphocyte depletion is associated with depressed cell mediated immunity leading to anergic skin test responses and impaired homograft rejection, there does not seem to be an unduly high incidence of opportunistic infections (Strober et al., 1967) and there are no reports of complicating malignancy.

\section{Case report}

A 40-year-old man first developed asymmetrical peripheral oedema at the age of 12 years. Extensive viral warts appeared at the age of 18 years and in 1966 , at 29 years, he presented with a right-sided pleural effusion, peripheral oedema and hypoalbuminaemia apparently due to excess protein loss from the gut consequent upon intestinal lymphangiectasia. At that time the absolute lymphocyte count was $0.8 \times 10^{9} / 1$ and serum albumin $27 \mathrm{~g} / 1$. A low fat diet and diuretics brought about complete resolution of the oedema and restoration of the serum albumin to within the normal range. The peripheral lymphocyte count remained low and the warts on the hands and feet gradually became more extensive, but he remained otherwise asymptomatic for 10 years when, in 1976, he presented with subacute small bowel obstruction. On examination he was thin and had a distended tympanitic abdomen with hyperactive bowel sounds and extensive warts on the hands and feet (Figs. 1 and 2). There was no peripheral oedema. The plasma proteins, full blood count, ESR, immunoglobulins, serum $\mathbf{B}_{12}$, serum folate, and liver function tests were normal. The absolute lymphocyte count varied between 0.6 and $1.0 \times 10^{\circ} / 1$ (mean $0.4 \times 10^{\circ} / 1$ ) and the plain X-ray abdomen confirmed small bowel obstruction. This obstruction failed to respond to conservative measures and at laparotomy 1 litre of chylous ascites was found together with gross thickening and hypomotility of the proximal small bowel. The serous surface of the bowel was leaking clear fluid; the mucosa was studded with lymphatic cysts. A midjejunal tumour had narrowed the lumen and there was a discrete ulcer proximal to it. A local resection and end-to-end anastomosis was performed.

After recovery from the immediate effects of operation a course of radiotherapy to the abdomen was given, but obstruction persisted with evidence of peritonitis. Parenteral nutrition was required but he finally developed more extensive abdominal sepsis with bacteraemia and died. It was of interest that despite this downhill course the warts almost completely disappeared from his hands (Fig. 3).

\section{Pathology}

The operative specimen consisted of a $13-\mathrm{cm}$ segment of jejunum. There was an annular stricture $6 \mathrm{~cm}$ from the distal end and $4 \mathrm{~cm}$ proximal to this there was a sharply defined ulcer measuring $2.5 \times 1.5$ $\mathrm{cm}$. The mucosal surface of the whole segment showed multiple 2-3 mm diameter polypoid lesions and the muscle was dark brown in colour (Fig. 4).

All the histological sections examined showed the features of intestinal lymphangiectasia (Whitehead, 1973; Fig. 5). The polypoid lesions were found to be 


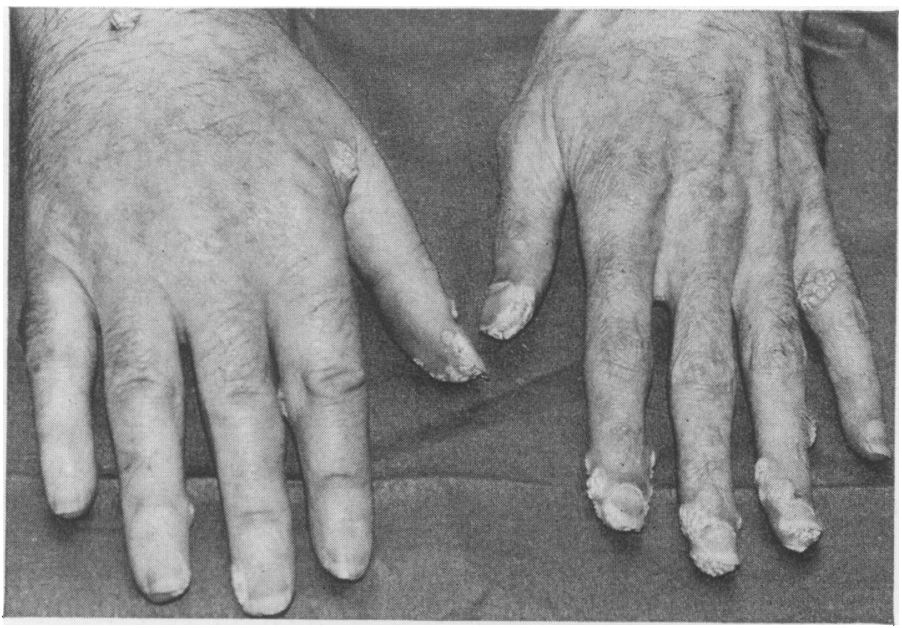

FIG. 1. The hands showing extensive wart formation.

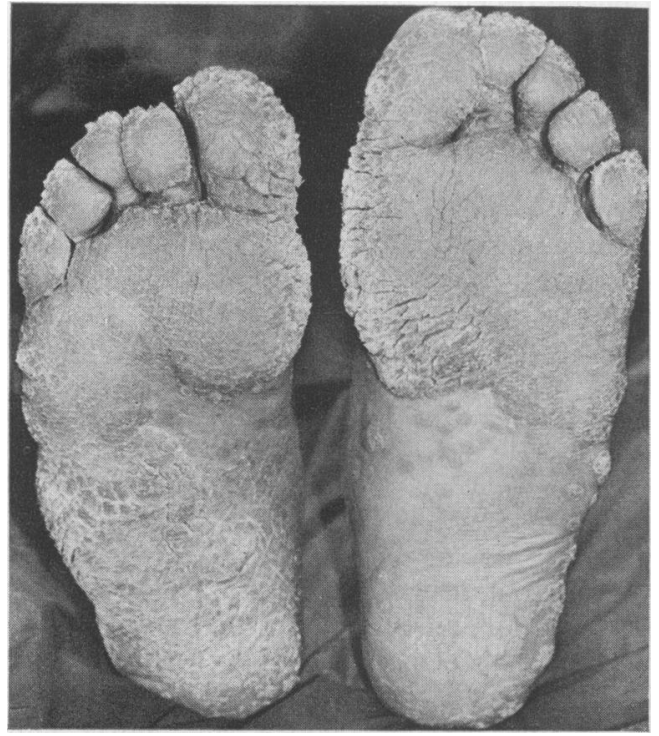

FIG. 2. The feet showing extensive wart formation.

large mucosal lymphoid follicles (Fig. 6). Both the stricture and the ulcer were the result of infiltration by a poorly differentiated lymphocytic lymphoma (Fig. 7). The muscularis propria showed marked atrophy with replacement fibrosis and accumulation of lipofuscin pigment within muscle fibres and in macrophages.

At the request of the relatives a post-mortem was limited to the abdominal cavity. A diffuse suppura- tive peritonitis with a loculated subphrenic abscess were found but no evidence of perforation. No further deposits of lymphoma were found either in the gastro-intestinal tract or in abdominal lymph nodes. There was lymphangiectasia of the duodenum $\mathrm{O}$ jejunum and ileum. The spleen weighed $140 \mathrm{~g}$ and showed a marked reduction in the size of the lymphoid follicles.

\section{Discussion}

Burnet (1957) first proposed that somatic mutations with their associated potential for neoplasia were kept in check by an immunological-surveillance mechanism. It was subsequently suggested by Thomas (1959) that homograft rejection might be an incidental effect of a defence system whose primary function was the control of neoplasia. This concept was supported by the appearance of various malignancies principally lymphomas in the recipients of renal transplants whose immune responsiveness had been suppressed with drugs and anti-lymphocyte serum (Penn et al., 1969; Schneck and Penn, 1971). Immunosuppressive therapy given for other conditions does not seem to carry the same high risk of malignancy and other explanations may account for development of these malignancies (Leibowitz and Schwartz, 1971). Other evidence supporting the role of immune defects in the production of malignancy is the increased incidence of lympho-reticular neoplasia in primary immune deficiency syndromes particularly those in which there is an element of defective cell-mediated immunity (Gatti and Good, 1971).

Strober et al. (1967) described eighteen cases of 


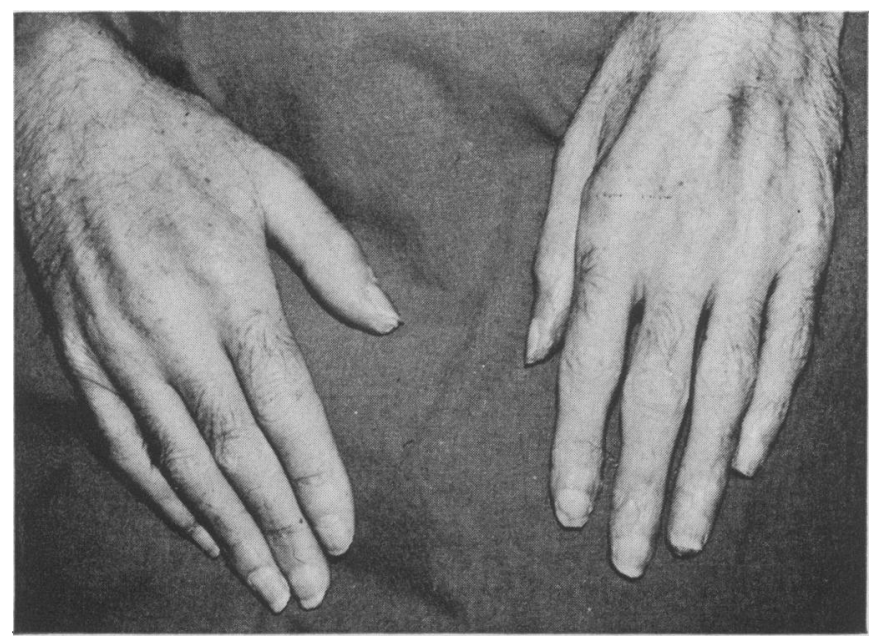

FIG. 3. The hands post-operatively and approximately one week before death showing disappearance of warts.

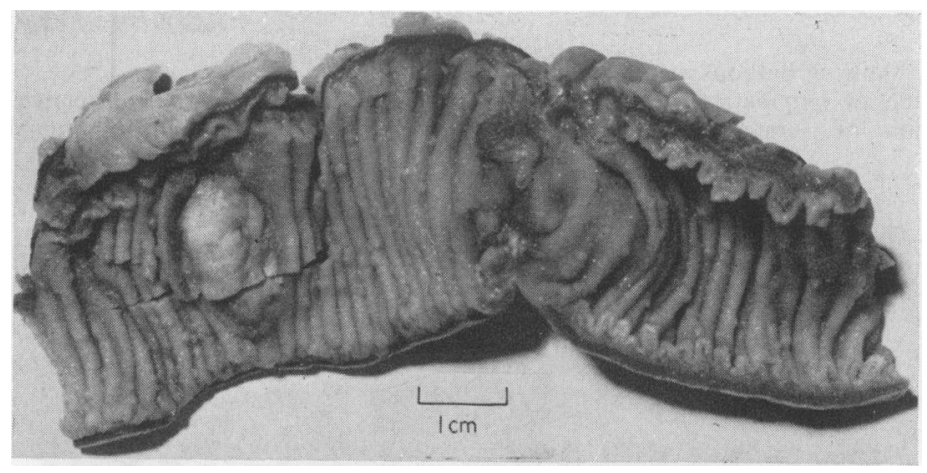
FIG. 4. Surgical specimen to show the annular stricture and proximally-situated
ulcer.

lymphangiectasia in whom there was a mean absolute peripheral lymphocyte count of $0.7 \pm 0.3 \times$ $10^{\circ} / 1$ (controls $2.5 \pm 0.6 \times 10^{\%} / 1$ ) and demonstrated profound depression of cellular immunity in that skin grafts were readily accepted. The mean lymphocyte count in the present patient $\left(0.4 \times 10^{9} / 1\right)$ thus places him at the lower end of the range of lymphocytopenia reported in other patients with lymphangiectasia. The authors did not investigate cell-mediated immunity in this patient because of various extraneous factors which would in themselves depress immune function and make interpretation impossible, including the recent operation, the presence of gross debility and malnutrition, and the use of radio- therapy. There seems little doubt, however, that this patient did have a marked depression of cellmediated immunity, that this was of primary importance in the production of the gross viral warts of the hands and feet, and that it probably contributed to the development of the small bowel lymphoma. The suggestion that chronic antigenic stimulation by frequent viral infections might be a factor in the production of malignancy in immune-deficient patients (Ten Besel, Studlan and Krivit, 1966) is of obvious relevance to this patient. The reason for the remarkable disappearance of this patient's warts despite the overall deterioration is uncertain. One possible factor is that during the course of his illness 


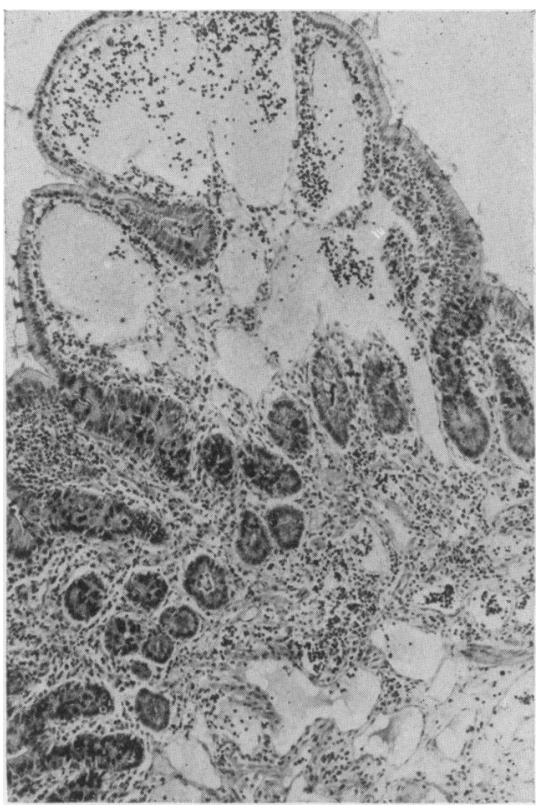

Fig. 5. Jejunal biopsy showing bulbous distortion of a villus due to distension of lymphatics in the lamina propria and dilatation of submucosal lymphatics. $(\mathrm{HE}, \times 24$.)

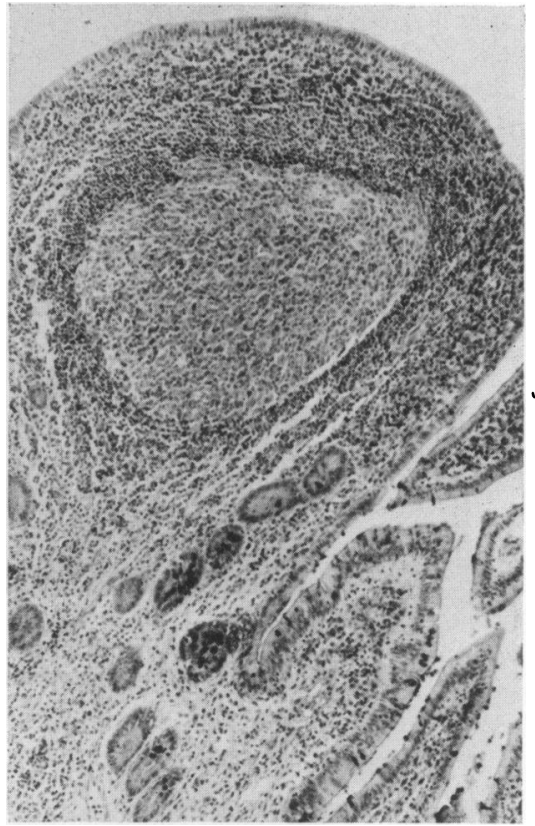

Fig. 6. Jejunal villus distended by a large lymphoid follicle with a prominent germinal centre. $(\mathrm{HE}, \times 24$.)

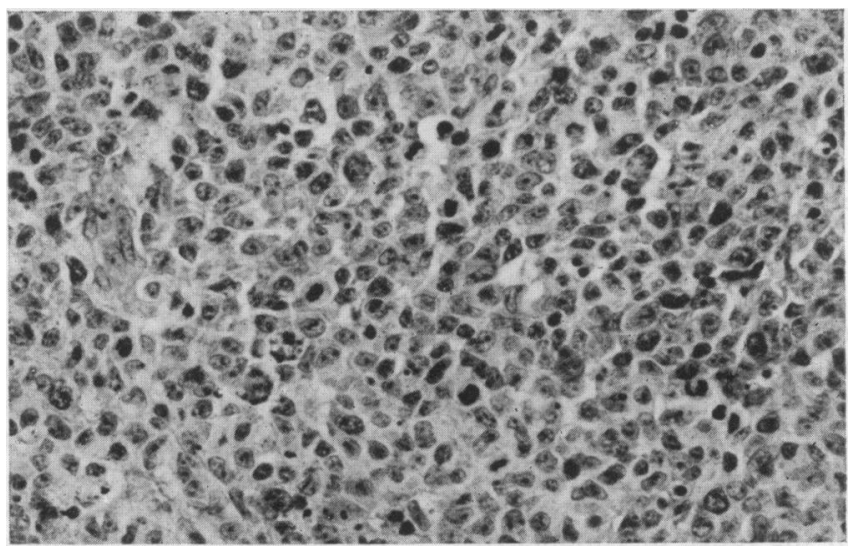

FIG. 7. Tumour deposit composed of sheets of poorly-differentiated lymphoblasts and including occasional giant cells and numerous mitotic figures. (HE, $\times 60$.) 
he received several blood transfusions which may have provided sufficient lymphocytes to mount an attack against the warts. The marked depletion of peripheral lymphocytes in this patient was in contrast to the maintenance of normal serum proteins, including immunoglobulins. This is of interest in view of the close similarity of hyperplastic lymphoid follicles (Fig. 6) with the nodular lymphoid hyperplasia found in hypogammaglobulinaemia (Hermans et al., 1966) and which is known to be associated with infection with Giardia lamblia.

Despite the marked depression of immune function in patients with lymphangiectasia opportunistic infections do not appear to be unduly common (Strober et al., 1967) and the authors have been unable to find any other reports of malignancy complicating this condition. It is perhaps the severity of their patient's lymphocyte depletion which has accounted for the appearance of such gross viral warts and ultimately to the complication of small intestinal lymphoma.

\section{References}

BURNET, F.M. (1957) Cancer-a biological approach. British Medical Journal, 1, 779.

GATTI, R.A. \& GooD, R.A. (1971) Occurrence of malignancy in immunodeficiency diseases. Cancer, $28,89$.

Hermans, P.E., Huizenga, K.A., Hoffman, H.N., Brown,
A.L. \& MARKowITz, H. (1966) Dysgammaglobulinaemia associated with nodular lymphoid hyperplasia of the small intestine. American Journal of Medicine, 40, 78.

Leibowitz, S. \& Schwartz, S. (1971) Malignancy as a complication of immunosuppressive therapy. In: Advances in Internal Medicine (Ed. by G. H. Stollerman). Year Book Medical Publishers, 17,

Penn, I., Hammond, W., Brettschneider, L. \& Starzl, T.E. (1969) Malignancy lymphoma in transplantation patients. Transplantation Proceedings, 1 (1), 106.

Roberts, S. \& Douglas, A.P. (1976) Intestinal lymphangiectasia: A study of five cases. Quarterly Journal of Medicine, $177,39$.

Ross, J.D., Reid, K.D.G., Ambujakshank, V.P., KInloch, J.P. \& SIRCUS, W. (1971) Recurrent pleural effusion, protein-losing enteropathy, malabsorption and mosaic warts associated with generalized lymphatic hyperplasia. Thorax, 76, 119.

SChNeCK, S.A. \& PenN, I. (1971) 'De Novo' brain tumour in renal transplant patient. Lancet, i, 983.

Strober, W., Wochner, R.D., Carbone, P.P. \& Waldmann, T.A. (1967) Intestinal lymphangiectasia: a protein-losing enteropathy with hypogammaglobulinaemia, lymphocytopenia and impaired homograft rejections. Journal of Clinical Investigation, 46, 1643.

Ten Besel, R.W., Studlan, E.M. \& Krivit, W. (1966) The development of malignancy in the course of the Aldrich syndrome. Journal of Pediatrics, 68, 769.

ThомAs, L. (1959) Reaction to homologous tissue antigens in relation to hypersensitivity. In: Cellular and Humoral Aspects of the Hypersensitive State (Ed. by H. S. Laurence), p. 529. Paul B. Hoeber, New York.

WhiteheAD, R. (1973) In: Mucosal Biopsy of the Gastrointestinal Tract, p. 116. W. B. Saunders Co. Ltd.

\title{
Quadriceps myositis: an appraisal of the diagnostic criteria of quadriceps myopathy
}

\author{
P. D. MOHR \\ B.Sc., M.B., Ch.B., M.R.C.P. \\ T. G. KNOWLSON \\ M.B., Ch.B., M.R.C.Path. \\ Departments of Neurology and Neuropathology, University of Manchester, Manchester
}

\begin{abstract}
Summary
A patient with the clinical picture of quadriceps myopathy was shown to have an underlying myositis. Review of the literature showed myositis to be a rare cause of the 'quadriceps syndrome' but it is important to identify this group because they are potentially treatable with corticosteroids.

\section{Introduction}

Selective wasting and weakness of the quadriceps muscles has been reported in two patients with
\end{abstract}

myositis (Denny-Brown, 1939; Turner and Heathfield, 1961), two patients with unspecified myopathy (Bramwell, 1922), twelve patients with muscular dystrophy (Erb, 1891; Walton, 1956; Mümenthaler et al., 1958; Wijngaarden et al., 1968; Espir and Matthews, 1973) and one patient with chronic spinal muscular atrophy (Boddie and Stewart-Wynne, 1974). The latter report suggested that quadriceps myopathy is a clinical syndrome with various underlying pathologies. A further patient with quadriceps wasting due to a myositis is now reported and the 